\title{
Characterization of Pulmonary Arterial Hypertension Using Right Ventricular Regional Curvedness Derived From CMR Imaging
}

\author{
Soo-Kng Teo ${ }^{*}$, Xiaodan $\mathrm{Zhao}^{2}$, Ru-San Tan ${ }^{2,3}$, Liang Zhong ${ }^{2,3}$, Yi Su ${ }^{1}$ \\ ${ }^{1}$ Institute of High Performance Computing, A*STAR, Singapore \\ ${ }^{2}$ National Heart Centre Singapore, Singapore \\ ${ }^{3}$ Duke-NUS Medical School, National University of Singapore
}

\begin{abstract}
Pulmonary arterial hypertension (PAH) is a hemodynamic and pathophysiological condition defined as mean pulmonary artery pressure greater than $25 \mathrm{mmHg}$ at rest by right heart catheterization (RHC). PAH may develop decompensated hemodynamics leading to progressive right ventricular $(R V)$ dilation, shape alteration, dysfunction and failure. However, quantification of $R V$ shape is challenging due to its complex geometry. We proposed using geometry based curvedness index at the end-systole phase $\left(C_{E S}\right)$ computed from cardiac magnetic resonance (CMR) imaging to characterize PAH patients. All participants underwent $C M R$ imaging and the $3 D R V$ geometry were reconstructed in the form of a triangulated surface mesh partitioned into 13 segments based on our previous works. Our preliminary results are: There is a significant difference in $C_{E S}$ for freewall segments aggregated across the basal, mid-layer and apical regions for the PAH and control groups $(p<0.05)$. For the mid-layer region, the mean $C_{E S}$ for free-wall segments for PAH patients with New York Heart Association (NYHA) class 2 is significantly different as compared to the control group ( $p<0.05)$. Similarly, for the apical region, the mean $C_{E S}$ for free-wall segments in $P A H$ patients in NYHA class 2 and 3 are also significantly different as compared to the control group ( $p<0.05)$.
\end{abstract}

\section{Introduction}

Pulmonary arterial hypertension (PAH) is one of the causes of right ventricle (RV) dysfunction due to pressure overload [1]. It is a serious and complex medical condition that is characterize by high blood pressure in the pulmonary arteries (the blood vessels that supply deoxygenated blood from the right side of the heart to the lung for oxygenation) due to both proximal and distal pulmonary artery (PA) remodeling [2]. This increased pulmonary arterial pressure leads to RV dilation (enlargement of the RV) through myocardial hypertrophy, increased passive stiffness and myofiber stress [3], and subsequent results in contractile dysfunction. From our previous patient-specific computational analysis, $\mathrm{PAH}$ can induce LV remodeling, and septum curvature alteration [3].

Clinical gold standard of diagnosis for PAH is performed using an invasive right heart catheterization (RHC), with PAH defined by a mean pulmonary arterial pressure greater than $25 \mathrm{~mm} \mathrm{Hg}$ at rest [4]. However, RHC is an invasive method and presents certain risk factors such as allergic reactions, bleeding, infection, bruising. Another more serious risk factor associated with RHC is the formation of blood clots that may potentially trigger cardiac arrest, stroke or other serious medical complications. Thus, the use of non-invasive imaging utilizing echocardiography and cardiac magnetic resonance (CMR) imaging for the assessment of PAH is increasingly been recognized $[5,6]$. However, the assessment of RV function other than the measurement of volumes and ejection fraction using CMR imaging is not as straight forward as compared to the left ventricle (LV) due to the non-regular RV shape. RV descriptors that are both sensitive and specific for the characterization of abnormal RV functions need to incorporate critical information including geometrical parameters, dilation and contraction [7]. Curvedness is a geometric property that can be quantified numerically and is being used as a descriptor of local heart function that does not require a frame of reference in contrast to other measures (e.g. longitudinal and radial strains). In our previous works, we have already applied the curvedness index to characterize LV functions in patients with the following conditions: hypertrophic cardiomyopathy [8], first-time myocardial infarction [9], ischemic dilated cardiomyopathy [10], and heart failure [11]. Similarly, we have also applied the curvedness index to characterize RV functions in patients with repaired tetralogy of Fallot [12].

In this study, we proposed using geometry based curvedness index at the end-systole $\left(\mathrm{C}_{\mathrm{ES}}\right)$ phase computed from the reconstructed 3D geometry of the RV surfaces derived from manual segmentation of cardiac magnetic 
resonance $(\mathrm{CMR})$ imaging to characterize $\mathrm{PAH}$ patients. To test our hypothesis, we compute the $\mathrm{C}_{\mathrm{ES}}$ for the $\mathrm{RV}$ based on CMR imaging and compared the aggregated $\mathrm{C}_{\mathrm{ES}}$ across the basal, mid-layer and apical regions between the PAH patients and healthy volunteers. Our objectives are two-fold: (i) first, we seek to demonstrate that the regional values of $\mathrm{C}_{\mathrm{ES}}$ between these two groups are different and (ii) the $\mathrm{C}_{\mathrm{ES}}$ index can be used as a proxy for correlating to the New York Heart Association (NYHA) functional class for PAH patients. The advantages of our approach are that the computation of the RV curvedness is automated and reproducible for a given set of input contours, thereby minimizing any intra- and inter- observer variabilities.

\section{Methods}

Two groups of subjects (23 normal controls and $23 \mathrm{PAH}$ patients) that are age- and sex-matched with left ventricular ejection fraction $>50 \%$ were prospectively recruited for this study. All subjects also gave informed consent for their participation in this study. The design of the study protocol was approved by the local Institutional Review Board.

\subsection{Cine MRI}

The CMR scan was performed using steady-state free precession (SSFP) cine gradient echo sequences on a 3.0T magnetic resonance scanner (Ingenia, Philips Healthcare, Netherlands) with a dStream Torso coil (maximal number of channels 32). Typical parameters used were: TR/TE $3 / 1 \mathrm{~ms}$, flip angle $45^{\circ}$, slice thickness $8-10 \mathrm{~mm}$, field of view $320 \mathrm{~mm}$ with in-plane spatial resolution of $<1.5 \mathrm{~mm}$. Standard long axis planes covering the ventricular two-, three-, four-chamber views and short-axis planes covering both ventricles (with 12-14 equidistant slices) were acquired in a single breath hold, with 30 temporal phases per cardiac cycle.

\subsection{Reconstruction of 3D RV model}

The approach for the 3D geometrical model reconstruction of the RV has already been described in our previous works [12, 13]. Briefly, the reconstruction approach can be summarized into the following steps: (i) Endocardial contours corresponding to the RV surface at the end-systole phase of the cardiac cycle are manually segmented using the CMRtools suite (Cardiovascular Solution, UK), (ii) Perform up-sampling of the RV endocardial contours to achieve a smooth reconstructed 3D surface, due to the relatively large spacing between the CMR image slices, (iii) Generate a dense set of points representing the RV endocardial surface using both the segmented and up-sampled RV contours, and (iv) Triangulate this dense set of points into a unstructured triangle mesh representing the 3D RV geometry using our in-house meshing code (Cardiowerkz). For analysis, the $\mathrm{RV}$ mesh is partitioned into 13 segments that are consistent with the standard 16-segment American Heart Association nomenclature based on our previous work [12].

\section{Results and Discussion}

The curvedness for all $13 \mathrm{RV}$ segments were computed from the $3 \mathrm{D}$ reconstructed $\mathrm{RV}$ geometry. We compared the aggregated $\mathrm{C}_{\mathrm{ES}}$ for both free-wall and septal segments across the basal, mid-layer and apical regions between the normal control and PAH patients. Based on the nomenclature (refer to Figure 3 in reference [12]) used, the free-wall segments correspond to segments 1-3 (basal region), segments 6-8 (mid-layer region) and segments 1112 (apical region). Similarly, the septal segments correspond to segments 4-5 (basal region), segments 9-10 (mid-layer region) and segment 13 (apical region). We observed that there is a significant difference in $\mathrm{C}_{\mathrm{ES}}$ for free-wall segments across the basal, mid-layer and apical regions for the PAH and control groups (Refer to Figure 1 left panel; $p<0.05)$. Specifically, $\mathrm{C}_{\mathrm{ES}}$ for normal controls are significantly higher as compared to PAH patients. This
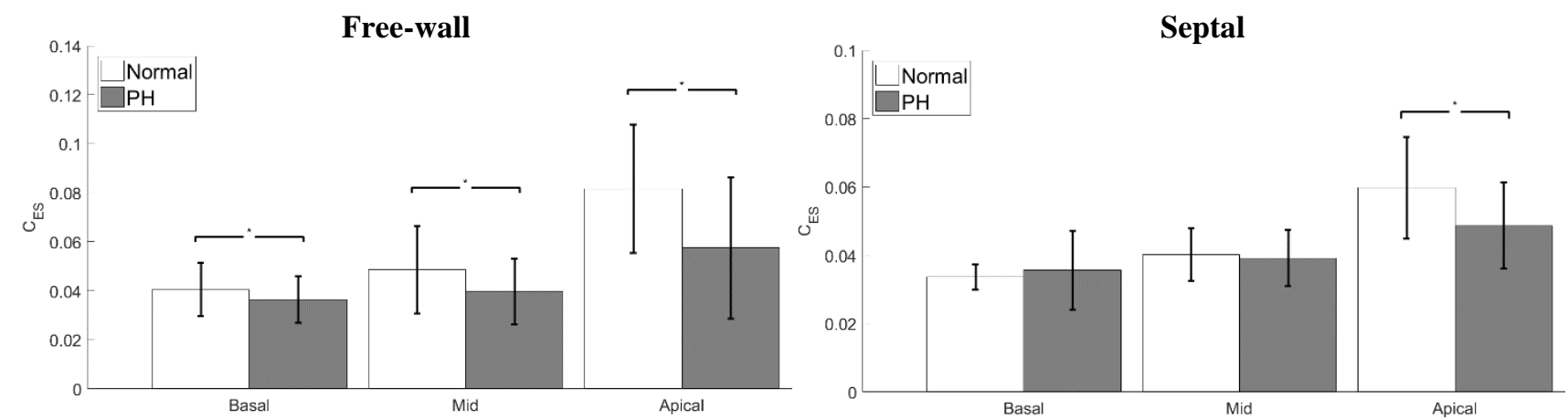

Figure 1. Comparison of $\mathrm{C}_{\mathrm{ES}}$ (mean \pm standard deviation) for free-wall segments (left panel) and septal segments (right panel) aggregated across the basal, mid-layer and apical regions $(* \mathrm{p}<0.05)$. Refer to main text for further discussion. 

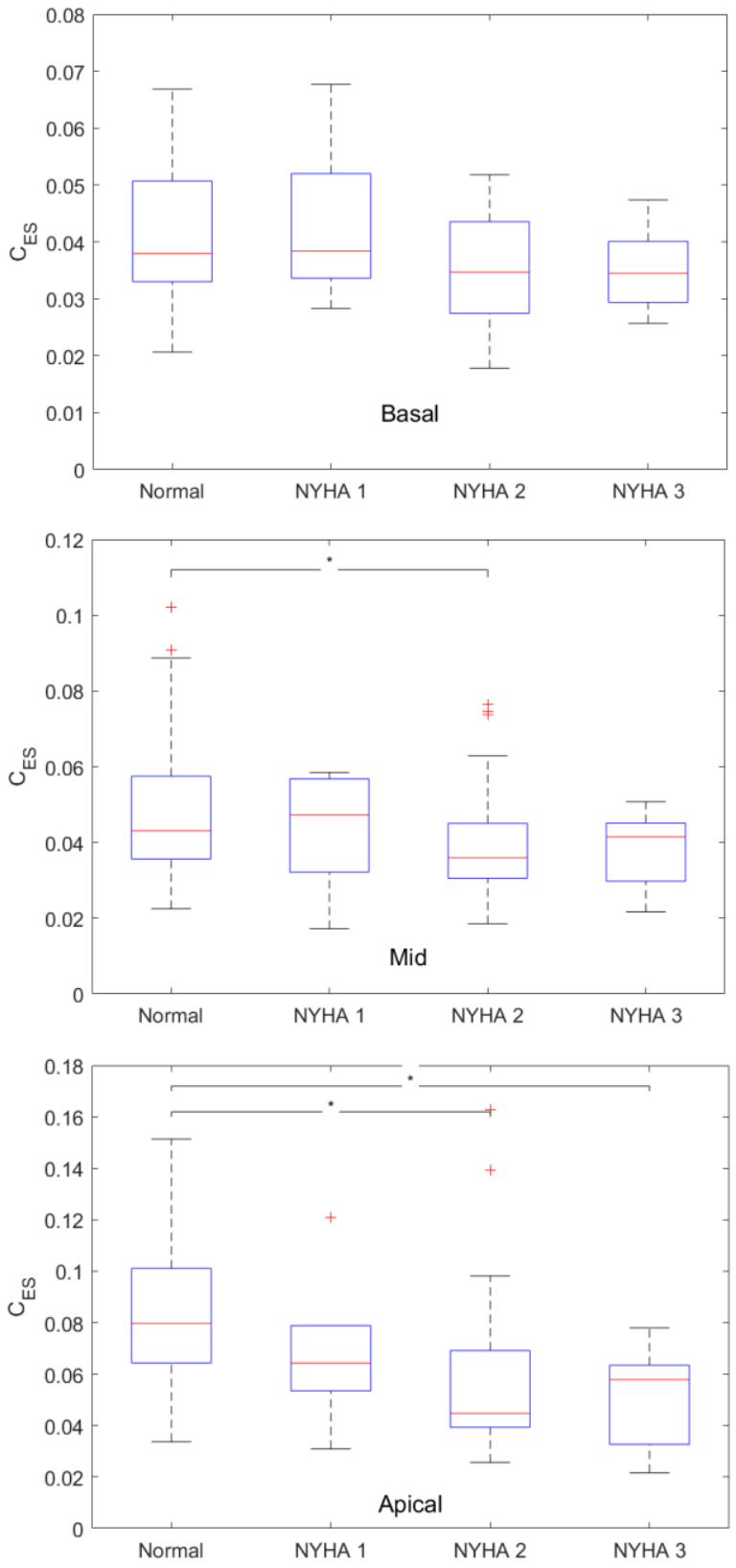

Figure 2. Box-whisker correlating $\mathrm{C}_{\mathrm{ES}}$ for free-wall segments (basal region - top panel, mid-cavity region middle panel and distal region - bottom panel) with the NYHA class.

trend was not observed in $\mathrm{C}_{\mathrm{ES}}$ for septal segments across the basal, mid-layer regions (refer to Figure 1 right panel). Only the $\mathrm{C}_{\mathrm{ES}}$ for septal segments in the apical regions shown significant difference between the two groups.

We postulated that the reduced $\mathrm{C}_{\mathrm{ES}}$ for free-wall segments in $\mathrm{PH}$ patients as compared to normal controls is an indication of the RV contractile dysfunction and can be used as a proxy to measure the degree of this contractile dysfunction. As the contractile function of the RV degenerates, we expect the RV shape at end-systole to lose its curvature as compared to normal controls since the myocardial contraction in the free-wall is reduced, thereby reducing the pumping capacity of the RV. This is further supported by the reduction in the RV ejection fraction (EF) for PAH patients as compared to normal controls. Furthermore, we also observed that the RV EF is further reduced in PAH patients who are classified in NYHA functional class 2 and 3 as compared to patients who are classified as NYHA functional class 1 . The RV EF (mean \pm standard deviation) as computed from our 3D model for both groups are: $70.1 \pm 5.7 \%$ (Normal controls, $\mathrm{N}=23$ ); $64.5 \pm 7.7 \%$ (PAH patients with NYHA class $1, \mathrm{~N}=3$ ); $52.7 \pm 17.1 \%$ (PH patients with NYHA class $2, \mathrm{~N}=15$ ) and $45.2 \pm 9.1 \%$ (PH patients with NYHA class $3, \mathrm{~N}=5$ ). For the septal segments, we postulate that the increased pulmonary arterial pressure has not resulted in significant LV contractile dysfunction in the PAH patients that were recruited for this study since all patients recruited have LV $\mathrm{EF}>50 \%$. There was only a significant difference in $\mathrm{C}_{\mathrm{ES}}$ for septal segments in the apical regions with PAH patients exhibiting reduced $\mathrm{C}_{\mathrm{ES}}$ as compared to normal controls. One potential hypothesis is that the thinner myocardium at the LV apical regions (corresponding to the RV septal segment 13) is more sensitive to the damage from the increased pulmonary arterial pressure and hence will be the first segment in the septal to develop contractile dysfunction. However, this hypothesis will need to be validated in a larger cohort of PAH patients with significantly reduced LV EF.

To further refine our analysis for PAH patients, we correlate $\mathrm{C}_{\mathrm{ES}}$ for free-wall segments to the NYHA functional class (refer to Figure 2). For the mid region, the mean $\mathrm{C}_{\mathrm{ES}}$ for free-wall segments for PAH patients in NYHA class 2 is significantly different as compared to the control group $(p<0.05)$. Similarly, for the apical region, the mean $\mathrm{C}_{\mathrm{ES}}$ for free-wall segments in PAH patients in NYHA class 2 and 3 are also significantly different as compared to the control group ( $\mathrm{p}<0.05)$. This analysis suggests that $\mathrm{C}_{\mathrm{ES}}$ may potentially be used as an additional proxy for the classification of PAH patients into NYHA functional class.

\section{Limitations}

Our approach for computing $\mathrm{C}_{\mathrm{ES}}$ is based on the manual segmentation of the RV endocardial surface from the CMR images which is time-consuming and may be subjected to potential intra- and inter- observer variabilities. However, it must be noted that our approach is fully automated and reproducible for a given set of segmented RV contours. In addition, the sample size of the current study is relatively small (23 patients) and the statistical powers of our analysis will have to be further validated in a larger sample size. Also, our current analysis is also limited to the RV 
without consideration of the LV. For PAH patients, the contractile dysfunction in the RV could potentially also lead to LV dilation and remodeling. Hence, it will be important to consider the contribution of the LV in subsequent analysis and to examine the interaction between contractile dysfunction developing in both ventricles.

\section{Conclusions}

We have described a computational approach to compute $\mathrm{C}_{\mathrm{ES}}$ for the $\mathrm{RV}$ from the reconstructed $3 \mathrm{D}$ geometry of its surfaces derived from manual segmentation of cardiac magnetic resonance imaging. Our results showed that there is a significant difference in $\mathrm{C}_{\mathrm{ES}}$ for free-wall segments across the basal, mid-layer and apical regions between the PAH and control groups. The mean $\mathrm{C}_{\mathrm{ES}}$ for free-wall segments in the mid-layer for $\mathrm{PH}$ patients in NYHA class 2 is also significantly different as compared to the control group. Similarly, for the apical region, the mean $\mathrm{C}_{\mathrm{ES}}$ for free-wall segments in PAH patients in NYHA class 2 and 3 are also significantly different as compared to the control group. In conclusion, $\mathrm{RV}$ free wall curvedness index derived from CMR images is significant reduced in PAH patients and may be a useful non-invasive imaging marker of $\mathrm{RV}$ dysfunction and severity of functional class.

\section{Acknowledgements}

This research was supported in part by research grants by the Singapore Ministry of Health's National Medical Research Council (NMRC/OFIRG/0018/2016, Zhong) and the Goh Cardiovascular Research Grant (Duke-NUSGCR/2013/0009, Zhong).

\section{References}

[1] Galiè N, Humbert M, Vachiery JL, Gibbs S, Lang I, Torbicki A, et al. 2015 ESC/ERS Guidelines for the diagnosis and treatment of pulmonary hypertension: The Joint Task Force for the Diagnosis and Treatment of Pulmonary Hypertension of the European Society of Cardiology (ESC) and the European Respiratory Society (ERS): Endorsed by: Association for European Paediatric and Congenital Cardiology (AEPC), International Society for Heart and Lung Transplantation (ISHLT). Eur. Heart J. 2016; 37(1):67-119.

[2] A. Zambrano BA, McLean NA, Zhao XD, Tan J-L, Zhong L, Figueroa CA, Lee LC, Beak S. Image-based computational assessment of vascular wall mechanics and hemodynamics in pulmonary artery hypertension patients. $\mathbf{J}$ Biomech 2018;68:84-92.

[3] B. Xi C, Latnie C, Zhao X, Tan J-L, Wall ST, Genet M, Zhong L, Lee LC. Patient-specific computational analysis of ventricular mechanics in pulmonary arterial hypertension. $\mathrm{J}$ Biomech Eng 2016;138(11).
[4] Hoeper MM, Bogaard HJ, Condliffe R, Frantz R, Khanna D, Kurzyna M, Langleben D, Manes A, Satoh T, Torres F, Wilkins MR, Badesch DB. Definitions and diagnosis of pulmonary hypertension. J Am Coll Cardiol 2013; 62:D4250.

[5] Milan A, Magnino C, Veglio F. Echocardiographic indexes for the non-invasive evaluation of pulmonary hemodynamics. J Am Soc Echocardiogr 2013; 23:225-239.

[6] Nogami M, Ohno Y, Koyama $\mathrm{H}$ et al. Utility of phase contrast MR imaging for assessment of pulmonary flow and pressure estimation in patients with pulmonary hypertension: comparison with right heart catheterization and echocardiography. J Magn Reson Imaging 2009; 30:973980.

[7] Voelkel NF, Quaife RA, Leinwand LA, Barst RJ, McGoon MD, Meldrum DR, Dupuis J, Long CS, Rubin LJ, Smart FW, Suzuki YJ, Gladwin M, Denholm EM, Gail DB; National Heart, Lung, and Blood Institute Working Group on Cellular and Molecular Mechanisms of Right Heart Failure. Right ventricular function and failure: report of a National Heart, Lung, and Blood Institute working group on cellular and molecular mechanisms of right heart failure. Circulation 2006; 114:1883-91.

[8] Zhao X, Tan RS, Tang HC, Teo SK, Su Y, Wan M, Leng S, Zhang JM, Allen J, Kassab GS, Zhong L. Left ventricular wall stress is sensitive marker of hypertrophic cardiomyopathy with preserved ejection fraction. Front Physiol. 2018; 9:250.

[9] Teo SK, Zhao X, Tan RS, Zhong L, Su Y. Comparison of left ventricular curvedness derived from CMR imaging with the wall motion score index for male patients after first-time myocardial infarction. Computing in Cardiology 2017; 44:71.

[10] Zhong L, Su Y, Yeo SY, Tan RS, Ghista DN, Kassab G. Left ventricular regional wall curvature and wall stress in patients with ischemic dilated cardiomyopathy. Am J Physiol Heart Circ Physiol 2009; 296:H573-H584.

[11] Zhong L, Su Y, Gobeawan L, Sola S, Tan R-S, Navia JL, Ghista DN, Chua T, Guccione J, Kassab GS. Impact of surgical ventricular restoration on ventricular shape, wall stress and function in heart failure patients. Am J Physiol Heart Circ Physiol 2011;300:H1653-H1660.

[12] Zhong L, Gobeawan L, Su Y, Tan JL, Ghista D, Chua T, Tan RS, Kassab G. Right ventricular regional wall curvedness and area strain in patients with repaired tetralogy of Fallot. Am J Physiol Heart Circ Physiol 2012; 302:H1306-H1316.

[13] Su Y, Tan ML, Teo SK, Lim CW, Zhong L, Tan RS Automatic generation of surface meshes for right ventricle with 1-to-1 correspondence from cine-MR images. Computing in Cardiology 2015; 42:9-1.

Address for correspondence.

Soo-Kng Teo.

1 Fusionopolis Way, \#16-16 Connexis, Singapore 138632

teosk@ihpc.a-star.edu.sg 